\title{
Cirugía laparoscópica e histeroscópica para embarazo ectópico sobreinfectado en cicatriz de cesárea previa: presentación de un caso
}

\author{
Catalina Agudelo R. ${ }^{1}$, Carlos Giovani Castro C. ${ }^{2}$ \\ ${ }^{1}$ Residente de Cirugía Endoscópica Ginecológica, Fundación Universitaria de Ciencias de la Salud. ${ }^{2}$ Fundación \\ Universitaria de Ciencias de la Salud - Hospital de San José, Bogotá. Colombia.
}

\section{RESUMEN}

El embarazo ectópico con implantación en la cicatriz de una cesárea previa es un evento muy raro a pesar de la alta tasa de cesárea a nivel mundial, el mecanismo fisiopatológico aún no se establece con claridad. Presentamos un caso de una paciente con diagnóstico de embarazo ectópico sobreinfectado en cicatriz de cesárea previa, tratada con resección quirúrgica de la lesión por histeroscopia y laparoscopia.

\section{PALABRES CLAVE: Embarazo ectópico, laparoscopia, cicatriz de cesárea, infección}

\section{SUMMARY}

Ectopic pregnancy implantation in a previous cesarean scar is a very rare condition despite the high caesarean rate worldwide. The pathophysiological mechanism is not yet clearly established. We present a case of a patient with an overinfected ectopic pregnancy, implanted in a previous cesarean scar treated with resection of the lesion by hysteroscopy and laparoscopy.

\section{KEY WORDS: Ectopic pregnancy, laparoscopy, cesarean scar, infection}

\section{INTRODUCCIÓN}

Con el aumento en la tasa de cesáreas para la atención del parto, se aumentan también los riesgos inherentes a esta intervención (1). El embarazo ectópico implantado en la cicatriz de una cesárea previa, aunque muy raro, constituye uno de estos riesgos. Este tipo de mala implantación favorece la ruptura uterina o la hemorragia de difícil control e impactan en la morbimortalidad materna (2). En 1978, Larsen y Solomon reportaron el primer caso de un embarazo implantado en la cicatriz de una cesárea previa (3), desde entonces varios casos han sido publicados (4), así como diferentes vías terapéuticas. El manejo médico para estas pacientes ha tenido éxito en algunos casos (5), sin embargo, con la realización de cirugía no solo se remueve el embarazo ectópico, sino que también se corrige el defecto uterino que favorecería una nueva implantación de un embarazo posterior en este sitio.

Describimos un caso de embarazo ectópico implantado en la cicatriz de una cesárea previa, sobreinfectado y manejado con laparoscopia e histeroscopia. 


\section{Caso clínico}

Paciente de 30 años con antecedente de embazo previo llevado a cesárea por desproporción céfalo pélvica 5 años antes. Consulta por malestar general y signos de respuesta inflamatoria sistémi$\mathrm{ca}$, al examen físico tacto vaginal normal, histerosonografía y resonancia magnética que reportan masa sólida dependiente de pared uterina anterior, ubicada a nivel ístmico hacia la izquierda, con límites mal diferenciados, de bordes irregulares, diámetro mayor de $3,5 \mathrm{~cm}$ (Figuras 1 a 4). Se realiza diagnóstico de embarazo ectópico sobreinfectado para el cual recibe tratamiento antibiótico de amplio espectro más legrado obstétrico. Cuatro semanas después la paciente continua con reactantes de fase aguda elevados, dolor pélvico y malestar general por lo que es llevada a histeroscopia diagnóstica donde se encuentra istmocele con diámetro mayor de $3 \mathrm{~cm}$ conteniendo masa mal definida, irregular, de aspecto necrótico e inflamatorio que invade miometrio en cara anterior. Se realiza resección de un segmento de la lesión, toma de biopsia y corrección de márgenes del defecto de cicatriz previa (Figura 5). Por la amplitud, la profundidad del defecto y la invasión trofoblástica al miometrio, se continua la resección de la masa por vía laparoscópica, logrando retirar todo el tejido comprometido (Figura 6). Se realiza histerorrafia con endosutura barbada en 2 planos (Figura 7). La paciente es dada de alta al día siguiente sin complicaciones. El reporte final de patología reporta escasas vellosidades coriales y células trofoblásticas con severos cambios degenerativos, abundante material fibrinohemorrágico y necrótico e infiltrado inflamatorio de neutrófilos.

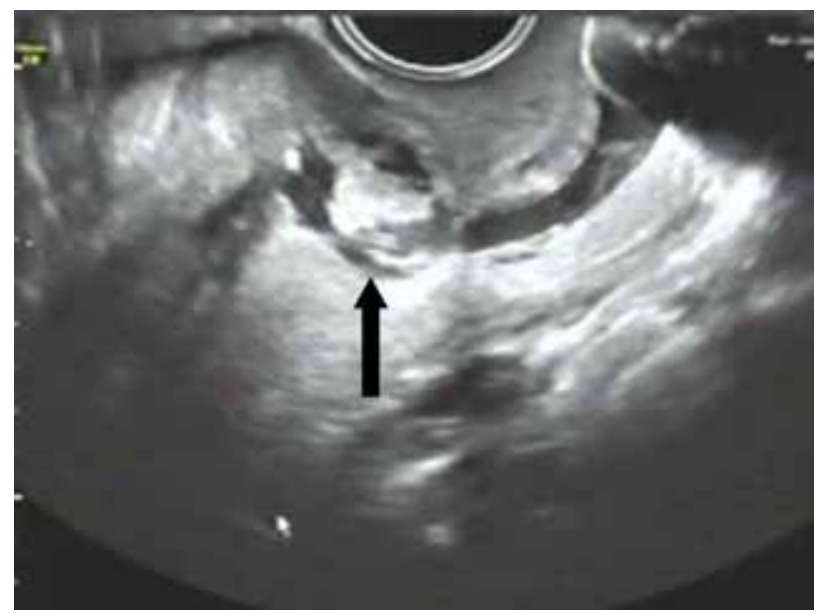

Figura 1. Histerosonografía. En corte longitudinal, se observa masa irregular en pared uterina anterior (flecha), que compromete cavidad endometrial.

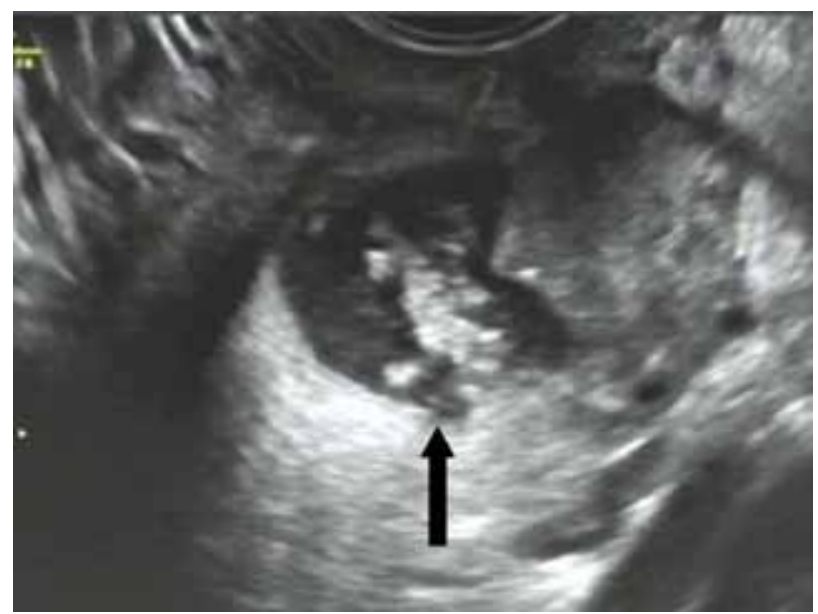

Figura 2. Histerosonografía. En corte transversal, se observa masa irregular en pared uterina anterior (flecha). 


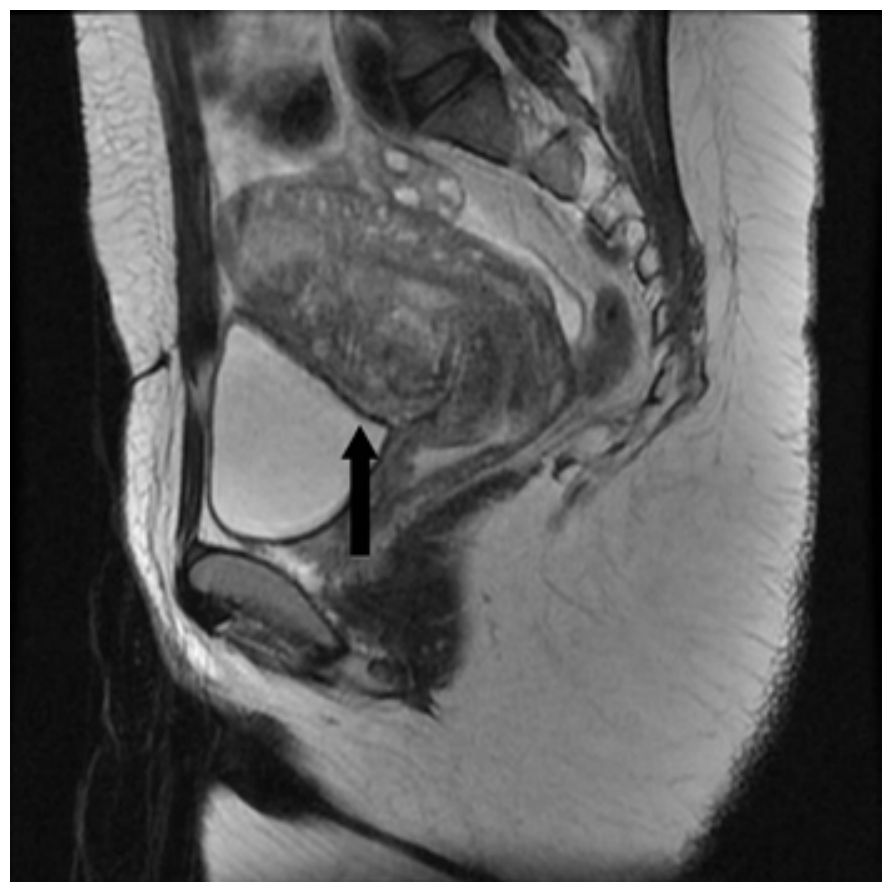

Figura 3. Resonancia magnética nuclear. En corte longitudinal, se observa masa irregular en pared uterina anterior (flecha).

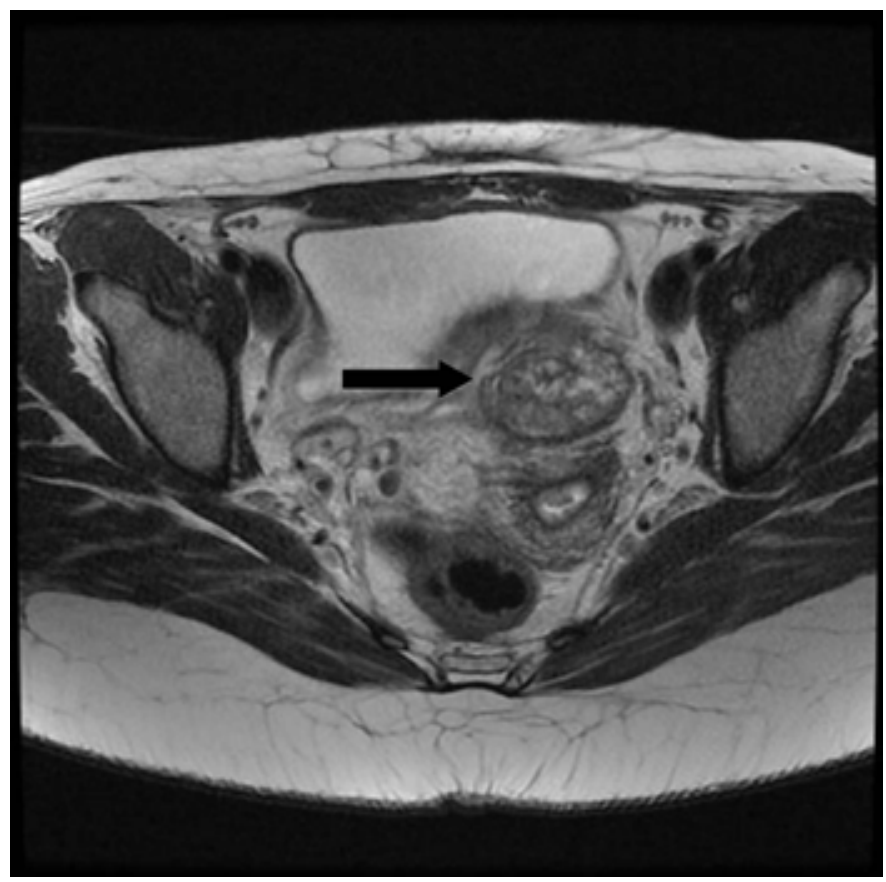

Figura 4. Resonancia magnética nuclear. En corte transversal, se observa masa irregular en pared uterina anterior (flecha). 


\section{ISTMOCELE}

\section{TROFOBLASTO}

Figura 5. Histeroscopia. Utilizando solución salina como medio de distensión, se observa defecto de pared uterina anterior (istmocele) ocupado por tejido irregular (trofoblasto).

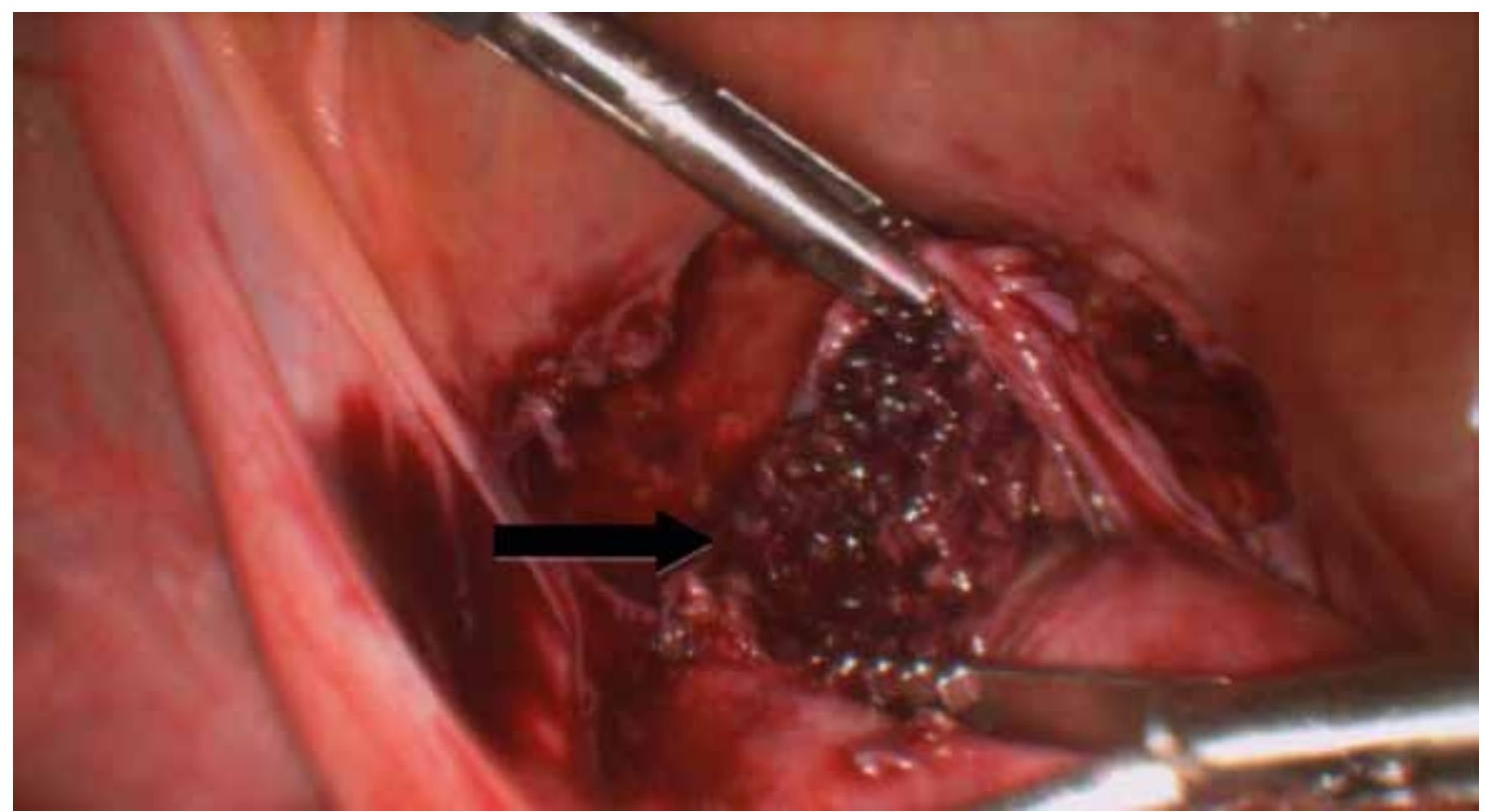

Figura 6. Laparoscopia. Se observa trofoblasto (flecha) que compromete pared uterina anterior en sitio de la cicatriz de la cesárea previa. 


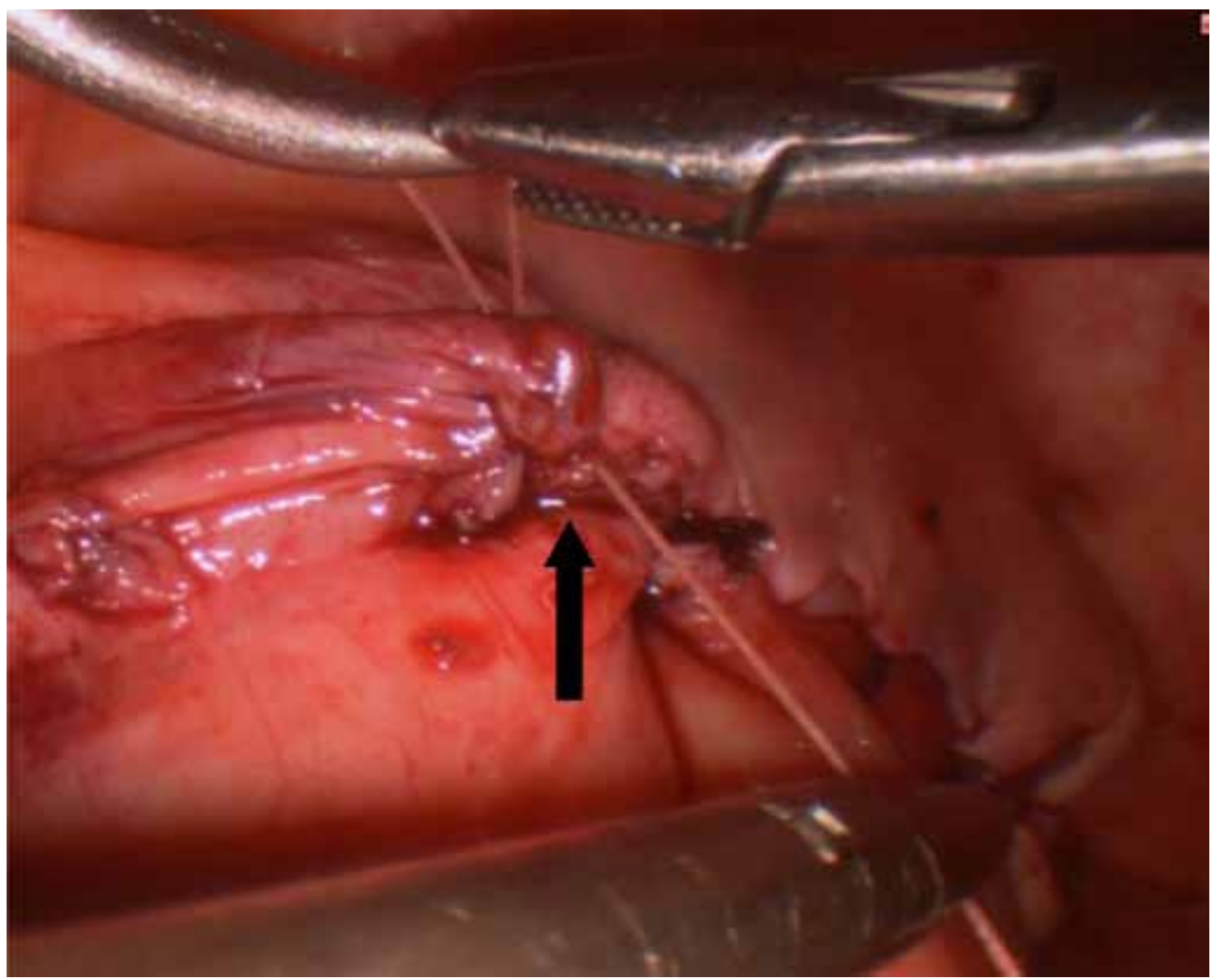

Figura 7. Laparoscopia. Se observa histerorrafia final luego resección total de embarazo ectópico ubicado en istmocele por defecto de la cicatriz de la cesárea previa.

\section{DISCUSIÓN}

El embarazo ectópico con implantación en la cicatriz de una cesárea previa es un evento muy raro a pesar de la alta tasa de cesárea a nivel mundial (4), el mecanismo fisiopatológico aún no se establece con claridad. Una hipótesis aceptada es la invasión del miometrio a través de un tracto tubular entre la cicatriz de la cesárea y el canal endometrial. Para realizar el diagnóstico debe existir sospecha clínica y presentarse una de las siguientes tres situaciones: el 39\% de los casos presenta sangrado vaginal escaso, sin dolor asociado; el $9 \%$ de los casos cursa con dolor abdominal leve aislado y el $16 \%$ de estas pacientes tiene ambos síntomas asociados.

La ecografía transvaginal es la herramienta de primera línea con una sensibilidad del $86,4 \%$, reporta cavidad uterina y canal cervical vacíos sin contacto con saco gestacional y detección en la pared anterior del istmo uterino del saco gestacional con presencia o no de embrión (6). Otros métodos de imagen o quirúrgicos como la resonancia magnética, ecografía tridimensional, histeroscopia o laparoscopia también son útiles en el diagnóstico (7).

No existe consenso sobre el manejo óptimo de esta entidad, depende del estado clínico de la paciente y la forma de presentación de cada caso. Hace algunos años la histerectomía era la opción más viable, sin embargo, ahora se prefiere un manejo menos radical con cirugía laparoscópica, histeroscópica o con manejo farmacológico como el metotrexate (8); éste último ha sido reportado con éxito en algunos casos (5). Existen escasos reportes en la literatura de la tasa de éxito del manejo conservador (9), por lo que no se recomienda en 
la actualidad, excepto en pacientes muy bien seleccionas con edad gestacional que no supere las 5 semanas.

Se encuentran diferentes series de casos de pacientes manejadas quirúrgicamente ya sea por vía laparoscopia o histeroscopia. Fuchs y cols (10), reportan 4 casos, uno de los cuales presentaba 11 semanas de gestación que hasta el momento es la edad gestacional más avanzada encontrada en la literatura con manejo laparoscópico, concluyendo en todos los casos con un abordaje seguro, eficaz y destacando el beneficio de la extracción total del tejido, la reconstrucción completa del defecto y el beneficio del seguimiento a corto plazo sin necesidad de extenderlo.

En cuanto al manejo histeroscópico Qing Yang y cols (11), evaluaron los resultados de este acceso quirúrgicos para el tratamiento del embarazo ectópico en la cicatriz de cesárea previa con 39 pacientes, 2 de las cuales solo requirieron una intervención adicional, concluyendo igualmente que esta vía es segura y factible para lograr éxito en el tratamiento. Otros autores reportan resultados interesantes con embarazos dobles utilizando histeroscopia de consultorio, la cual no requiere dilatación cervical y realiza bajo sedación consciente logrando la eliminación completa del embarazo ectópico (12).

Presentamos un caso único en la literatura de una paciente a la que realizamos ambas técnicas quirúrgicas conservadoras en el mismo tiempo quirúrgico, logrando la extracción completa del tejido del embarazo ectópico, foco de infección persistente, así como logrando la corrección total del defecto de la cicatriz, ambos procedimientos con excelentes resultados, disminuyendo para esta paciente el riesgo de embarazo ectópico recurrente en el mismo sitio del defecto y el riesgo de ruptura uterina en un embarazo eutópico posterior.

\section{REFERENCIAS}

1. Annappa M, Tripathi L, Mahendran M. Caesarean section scar ectopic pregnancy presenting as a fibroid. J Obstet Gynaecol. 2009;29(8):774.

2. Lee Ch, Wang Ch, Chang A, Yen Ch, Soong Y. Laparoscopic management of an ectopic pregnancy in a previous caesarean section scar. Hum Reprod. 1999;14(5):1234-6.

3. Larsen JV, Solomon MH. Pregnancy in a uterine scar sacculus-an unusual cause of postabortal haemorrhage. A case report. S Afr Med J. 1978;53(4):142-3.

4. Jiang S, Zhao S. Laparoscopic surgery for ectopic pregnancy within caesarean scar. Clin Exp Obstet Gynecol. 2013;40(3):440-4.

5. Deepti S, Usha MG, Krishnadas S. Successful treatment of a repeat caesarean scar ectopic pregnancy with transvaginal intraamniotic instillation of methotrexate. J Obstet Gynaecol India. 2013;63(3):209-10.

6. Fylstra DL. Ectopic pregnancy within a cesarean scar: a review. Obstet Gynecol Surv. 2002;57(8):537-43.

7. Phupong V, Narasethkamol A, Ultchaswadi P. Pregnancy in caesarean section scar. J Obstet Gynaecol. 2011;31(3):204-6.

8. MacKintosh M, Elkattan E, Mahendran M, Basama F. An asymptomatic ectopic pregnancy in a caesarean section scar. J Obstet Gynaecol. 2010;30(8):868-9.

9. Al-Hashimi S, Maiti S, Macfoy D. Successful conservative management of ectopic pregnancy in caesarean section scar. BMJ Case Rep. 2012;2012. pii:bcr2012006925.

10. Fuchs N, Manoucheri E, Verbaan M, Einarsson Jl. Laparoscopic management of extrauterine pregnancy in caesarean section scar: description of a surgical technique and review of the literatura. BJOG. 2015;122(1):137-40

11. Yang Q, Piao S, Wang G, Wang Y, Liu C. Hysteroscopic surgery of ectopic pregnancy in the cesarean section scar. J Minim Invasive Gynecol. 2009;16(4):4326.

12. Mollo A, Alviggi C, Conforti A, Insabato L, De Placido G. Intact removal of spontaneous twin ectopic caesarean scar pregnancy by office hysteroscopy: case report and literature review. Reprod Biomed Online. 2014;29(5):530-3. 